\title{
The delay in sharing research data is costing lives
}

\author{
Josh Sommer
}

\begin{abstract}
It is not uncommon for potentially life-saving research data to be published years after being generated. But the setback to progress caused by the delay in releasing data is troublesome for people who selflessly participate in trials and desperately await new therapies. Scientists need to feel greater urgency to share their findings quickly, and they need additional avenues to facilitate this process.
\end{abstract}

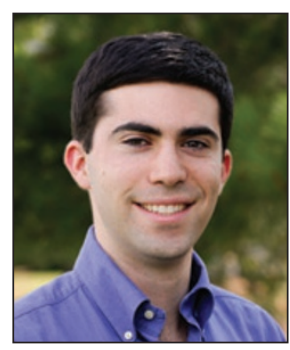

In 2006, as a freshman at Duke University, I was diagnosed with a rare form of bone cancer called chordoma. The disease has an average survival of seven years and a cure rate-through surgery and radiation-of approximately three in ten people. Unwilling to accept those statistics, and convinced that modern science could deliver a cure in time, my mother and I resolved to do everything in our power to outrun my disease.

Hoping to enlist in the fight against my cancer, I joined the lab of Michael Kelley, an oncologist at Duke, who happened to be the only federally funded chordoma researcher. Meanwhile, my mother, Simone Sommer, and I contacted dozens of other researchers who had published on chordoma or related topics to see what we could do to help. In our conversations with these researchers, we observed that progress was hindered in large part by infrequent communication and collaboration between labs. The need to overcome this and other obstacles ultimately drove us to start the Chordoma Foundation in 2007.

Over the past three years, the Chordoma Foundation has worked to systematically break down the barriers to developing better treatments. We have funded the development of cell lines and animal models and have facilitated sharing of tissue. We have convened two international chordoma research workshops, and we routinely connect researchers who have complementary interests, resources or information. All this has contributed to a recent uptick in discoveries, including the identification of several new potential therapeutic targets for chordoma.

Although this progress gives me hope, seeing friends and fellow chordoma patients become sickened and ultimately lose their battle with this tenacious disease is a constant reminder that time is of the essence. We must move faster. Giving researchers the resources and funding they need is within the power of the patient community-however, there is

a limit to the extent to which we can speed the flow of information between researchers.

Whereas some researchers graciously share unpublished data with colleagues, in the life sciences it is still the norm for data to be published years after it is generated (or sometimes not at all) and to be safeguarded until publication. A recent study of publications in 14 journals in fields of biology-granted, not medical fieldsfound a median submission delay (time from last data collection to submission of manuscript) of 189-696 days and a median publication delay (time from submission to publication) of $181-402$ days ${ }^{1}$. This is problematic, because developing treatments requires making a series of discoveries probably drawn from multiple labs. If, for the sake of argument, the key to unlocking a new treatment is just five sequential discoveries away, and, on average, one to three years go by before discoveries are transmitted between labs, then the path to a treatment could easily be delayed for ten years or more. Patients simply can't afford to wait for this protracted process to run its course.

Making science work fast enough for patients will require researchers to treat information with greater urgency. Surely, if anyone knew that he or she possessed life-saving data, he or she would act swiftly to share it, just as an intelligence officer would rush to report evidence of an impending terrorist attack. However, the intelligence that researchers gather about disease usually does not point directly to an immediate treatment or cure. Rather, each observation represents a clue, which, when combined with the observations from other labs, could potentially add up to a life-saving discovery. Individual investigators can't know the full value of their data to others, so they often don't demonstrate a sense of urgency to share it.

Without that sense of urgency, or other incentives for real-time communication, delays caused by circumstances within a lab or through the peer-review process may seem acceptable. For patients in desperate need of cures, and for funders of medical research (principally taxpayers) expecting a timely return on their investment, this delay is unacceptable.

Not only do scientists need to feel a sense of urgency to share data, they need more academically acceptable means to do so. The prevailing medium of scholarly communication-the peer-reviewed journal article-although valuable in many ways, was not designed as a tool to help in the inherently urgent task of curing disease.

One possible approach to facilitating rapid data sharing came in response to the worry generated by the H1N1 influenza pandemic. PLoS Currents: Influenza was created as a vehicle for speedy dissemination of ideas and findings in the field of influenza through an accelerated peer-review process. For anyone whose life is threatened by disease, the situation is no less dire. So why not treat information about other diseases with a commensurate level of urgency? Given that everyone will someday be touched by disease, it behooves us all to adopt new systems of biomedical information exchange that maximize the pace of discovery for all diseases while serving the needs of scientists and patients alike.

\section{Josh Sommer is executive director of the Chordoma Foundation. He launched the North Carolina-based foundation in 2007 with his mother, Simone Sommer}

1. O'Donnell, R.P., Supp, S.R. \& Cobbold, S.M. Conserv. Biol. 24, 615-620 (2010). 